EPJ Web of Conferences 19, 06006 (2012)

DOI: $10.1051 /$ epjconf/20121906006

(C) Owned by the authors, published by EDP Sciences, 2012

\title{
A new perspective on mapping the inner Galaxy
}

\author{
G. Zasowski ${ }^{1, a}$, R.A. Benjamin ${ }^{2}$ and S.R. Majewski ${ }^{1}$ \\ 1 University of Virginia \\ 2 University of Wisconsin, Whitewater
}

\begin{abstract}
Due to the very strong interstellar extinction in the inner Galaxy, the stellar complexities of this region have only recently begun to be explored, with many infrared surveys now taking advantage of the reduced extinction at longer wavelengths to study the geometry, chemistry, and dynamics of the Milky Way's innermost kiloparsecs. The new approach presented here applies the RJCE extinction correction method and the longitude-magnitude visualization technique to an extensive near+mid-infrared database (2MASS + GLIMPSE), and we assess the usefulness of this approach in exploring large-scale Galactic structure. By deriving physical parameters of the long bar and central bulge, we demonstrate this approach's efficacy in using archived data to trace large-scale structures previously only mapped by time-intensive, dedicated surveys.
\end{abstract}

\section{INTRODUCTION AND DATA}

Enabled by recent advances in infrared (IR) detection technology, much progress has been made in studying the distribution of stars in the Milky Way's heavily-extincted midplane and bulge regions. However, the census of large stellar components - including the bulge(s), bar(s), spiral arms, and rings - is far from complete. One of the most interesting questions concerns the so-called "long bar," whose orientation has proven surprisingly difficult to constrain, and whose kinematics and chemistry are largely unknown. This poster presents a preliminary study of the long bar and outer bulge using near- and midIR photometry from 2MASS and Spitzer GLIMPSE, combined with a stellar population model to aid in interpretation of the results.

We have applied extinction corrections using the Rayleigh-Jeans Color Excess method ([8]), and we use the longitude-magnitude visualization tool ([2]) to identify and trace stellar density fluctuations that extend coherently over many degrees of longitude.

\section{THE LONG BAR}

We detect the long bar of the Milky Way in the longitude-magnitude signature of red clump (RC; metalrich, helium-core-burning) giant stars. Due to the magnitude limits of 2MASS in the crowded midplane, we can only map the midplane long bar in uncorrected magnitudes, but we use Spitzer $4.5 \mu$ m data to minimize the effects of extinction.

Comparing recent measurements of the long bar angle shows that our best-fit angle of $\phi \sim 35^{\circ}$ is consistent within the uncertainties with many independent studies using a wide variety of measurement techniques (Figure 1, left).

\footnotetext{
a e-mail: gz2n@virginia.edu
}

This is an Open Access article distributed under the terms of the Creative Commons Attribution-Noncommercial License 3.0, which permits unrestricted use, distribution, and reproduction in any noncommercial medium, provided the original work is properly cited. 

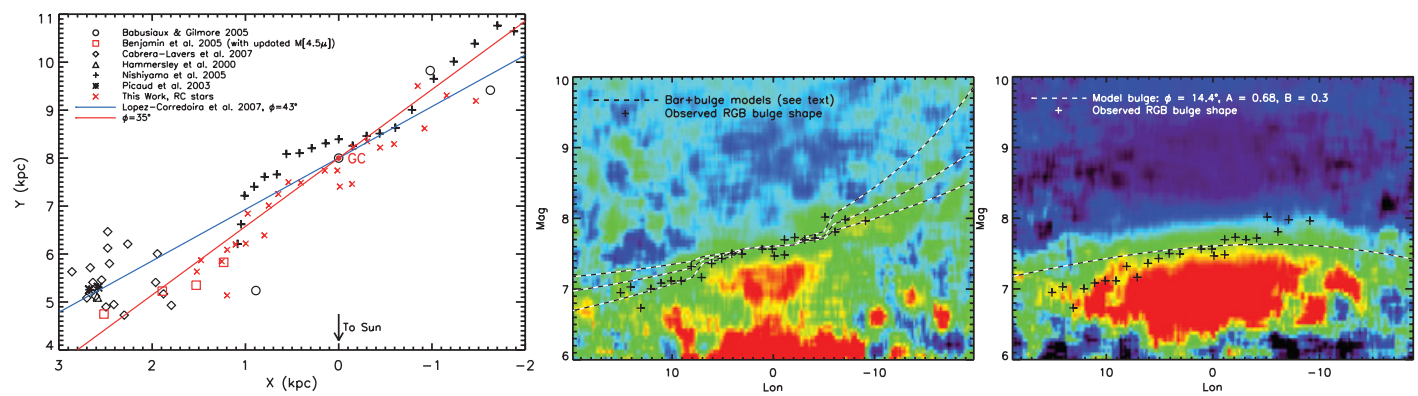

Figure 1. Left: We calculate a line-of-sight angle for the long bar of $\phi \sim 35^{\circ}$ and compare our measurements of the long bar's position with those of previous studies ([1-3, 6, 7, 9, 9, 10]). Center: A $4.5 \mu$ m longitude-magnitude plot showing the bulge RGB signature, overplotted with the observed RC points (plus signs, shifted to RGB magnitudes) and simple models of a bar only (large $|l|$ ), a bulge only (small $|l|$ ), and a bar + a bulge (intermediate $|l|$ ) as dashed lines, for bar angles of $10-50^{\circ}$. Right: Similar to the center panel but for the TRILEGAL model ([4]), overplotted with the GLIMPSE RC bar and bulge points (plus signs) and the density peaks for the model's triaxial bulge (dashed line).

Beyond $l \sim 27^{\circ}$, we find little-to-no detectable long bar signature, which places the bar end at a Galactic radius $R \sim 4.1 \mathrm{kpc}$, assuming $R_{\odot}=8.0 \mathrm{kpc}, \mathrm{M}_{4.5 \mu \mathrm{m}}(\mathrm{RC})=-1.58 \mathrm{mag}$, and $\phi=35^{\circ}$.

\section{THE BULGE}

In the Galactic bulge itself, we measure the magnitude of the peak stellar density as a function of longitude and wavelength for the extinction-corrected RGB stars. We find close similarity between the shape of the RGB longitude-magnitude feature and that of the RC stars, affirming that these populations reside in the same physical structure (Figure 1, center). We also confirm that the dereddened colors of the feature are consistent with middle-aged, approximately solar-metallicity RGB giants ([5]).

By the mismatch between the structure of the observed feature (Figure 1, center) and the bar-less TRILEGAL model (Figure 1, right), we demonstrate the impact of the bar on the star counts down to $|l| \sim 3^{\circ}$. The combination of bar and bulge model components appears to reproduce the observed feature shape well, whereas the TRILEGAL model would clearly be ill-fit by a similar combination of components.

\section{CONCLUSIONS}

We find the combination of RJCE extinction corrections and longitude-magnitude plots to be a potentially powerful tool for studying the large-scale stellar density of the inner Galaxy. Our best-fit orientation angle for the long bar is $35^{\circ}$; though this value is consistent (within the uncertainties) with previous results, the large amount of scatter in the measurements indicates that the picture is not yet complete. We also demonstrate that a bar component is required to reproduce the observed magnitude distribution, even within the bulge-dominated parts of the Galaxy.

\section{References}

[1] Babusiaux, C., \& Gilmore, G., MNRAS 358, (2005) 1309

[2] Benjamin, R. A., Churchwell, E., Babler, B. L., et al., ApJL 630, (2005) L149

[3] Cabrera-Lavers, A., Hammersley, P. L., González-Fernández, C., et al., A\&A 465, (2007) 825

[4] Girardi, L., Groenewegen, M. A. T., Hatziminaoglou, E., \& da Costa, L., A\&A 436, (2005) 895

[5] Girardi, L., Bertelli, G., Bressan, A., et al., A\&A 391, (2002) 195 
Assembling the Puzzle of the Milky Way

[6] Hammersley, P. L., Garzón, F., Mahoney, T. J., López-Corredoira, M., \& Torres, M. A. P., MNRAS 317, (2000) L45

[7] López-Corredoira, M., Cabrera-Lavers, A., Mahoney, T. J., et al., AJ 133, (2007) 154

[8] Majewski, S. R., Zasowski, G., \& Nidever, D. L., ApJ 739, (2011) 25

[9] Nishiyama, S., Nagata, T., Baba, D., et al., ApJL 621, (2005) L105

[10] Picaud, S., Cabrera-Lavers, A., \& Garzón, F., A\&A 408, (2003) 141 